\title{
Épidémiologie moléculaire de la rougeole : Quelle information nous apporte-t-elle et pourquoi est-ce important?
}

\author{
Hiebert $J^{1 \star}$ et Severini $A^{1}$ \\ Laboratoire national de microbiologie, Agence de la santé publique du Canada, Winnipeg (Manitoba) \\ * Correspondance à : Joanne.Hiebert@phac-aspc.gc.ca
}

\section{Résumé}

Contexte : L'épidémiologie moléculaire de la rougeole était un élément clé de la vérification de l'élimination de la rougeole indigène au Canada et constitue un outil précieux dans le cadre d'enquêtes de santé publique, à la fois pour établir s'il existe des liens entre les cas de rougeole simultanés et pour indiquer les sources possibles d'importation. Même s'il existe 24 génotypes distincts, le génotype n'est généralement pas suffisant pour décrire l'épidémiologie moléculaire complexe des cas de rougeole. On utilise la séquence génétique exacte des 450 derniers nucléotides du gène $(\mathrm{N}-450)$ de la nucléoprotéine $(\mathrm{N})$. Le génome de la rougeole mute très lentement et, donc, des cas dans la même chaîne de transmission ont généralement des séquences $\mathrm{N}-450$ identiques. Au Canada, le Laboratoire national de microbiologie séquence le gène N-450 et le dépose dans la base de données de l'OMS réservée aux séquences de rougeole et appelée MeaNS. Cette base de données peut servir à trouver d'autres zones géographiques où la séquence de la rougeole a été détectée, afin d'appuyer ou d'exclure d'éventuels liens. Pour les séquences N-450 couramment détectées, la base de données MeaNS désigne une " variante de la séquence ». Les variantes de la séquence servent de caractéristiques pour définir les cas de rougeole ayant des séquences identiques. Cette désignation est fondamentale pour décrire l'épidémiologie moléculaire de la rougeole à l'échelle locale et internationale. À mesure que l'éradication de la rougeole progresse dans le monde entier, la diversité génétique de la maladie diminue et les diverses importations de rougeole à partir d'un même réservoir peuvent s'avérer difficiles à différencier à l'aide des méthodes actuelles. II est nécessaire d'étendre les méthodes de séquençage au-delà du gène N-450. Même si le séquençage du gène de l'hémagglutinine $(\mathrm{H})$ dans son ensemble peut se révéler utile (ce qui est, à l'heure actuelle, régulièrement fait au Laboratoire national de microbiologie), le séquençage du génome entier sera nécessaire pour une surveillance moléculaire efficace en vue de surveiller l'élimination durable de la rougeole au Canada.

\section{Introduction}

Le Canada a éliminé la rougeole indigène. Cette réalisation est documentée dans un rapport soumis à l'Organisation panaméricaine de la santé (OPS)/Organisation mondiale de la Santé (OMS) [1]. Le dernier cas endémique de rougeole au Canada a eu lieu en 1997, et la rougeole a été éliminée de l'ensemble de la région des Amériques en 2002 (2). L'épidémiologie moléculaire de la rougeole est apparue comme un élément clé dans la vérification de l'élimination de la rougeole en démontrant l'absence d'un génotype endémique et continuera d'être essentielle dans la surveillance de l'élimination de la maladie. En outre, l'épidémiologie moléculaire de la rougeole est un outil précieux dans le cadre d'enquêtes de santé publique, à la fois pour établir s'il existe des liens entre les cas ou les éclosions de rougeole simultanés et pour indiquer les sources possibles d'importations. Cet article fournit des renseignements qui permettent de comprendre les données sur le génotypage de la rougeole, afin de démontrer l'utilité et la nécessité de l'épidémiologie moléculaire de la rougeole au Canada et d'offrir matière à discussion sur les orientations futures dans un cadre d'élimination. 


\section{Génotypage de la rougeole 101}

Un aspect important de la surveillance de la maladie est la capacité à différencier les lignées, les types ou les variantes d'un pathogène. Ce processus est généralement désigné sous le nom de typage et, historiquement, les pathogènes du même genre ou de la même espèce sont génotypés selon leurs caractéristiques phénotypiques, comme les marqueurs biochimiques ( $p$. ex. la présence ou l'absence de toxines) ou les marqueurs sérologiques (c.-à-d. les types d'antigènes présents sur le pathogène). Il s'agit en grande partie du processus utilisé pour les bactéries entériques (p. ex. la Salmonella et l'E. coli) et les virus de la grippe. Toutefois, pour un certain nombre de pathogènes, y compris la rougeole, il existe trop peu de différences phénotypiques pour employer cette méthode de typage. L'avènement de la technologie de séquençage de l'ADN (procédé de détermination de l'ordre et de l'identité des nucléotides dans une molécule d'ADN) a permis de déceler les zones des génomes permettant de différencier les lignées. II s'agit du processus de génotypage.

L'OMS a découvert 24 génotypes de la rougeole distincts sur le plan phylogénique et désignés sous les noms B1, B2, B3, C1, C2, D1, D2, D3, D4, D5, D6, D7, D8, D9, D10, D11, E, F, G1, G2, G3, H1 et H2. Les lettres désignent la principale variante génétique et les chiffres, les sous-clades (3). Les génotypes sont des regroupements de séquences génétiquement liées avec une variabilité inhérente, et la désignation du génotype ne suffit généralement pas pour décrire l'épidémiologie moléculaire complexe des cas de rougeole. Par conséquent, l'OMS recommande d'utiliser, au minimum, la séquence (ou variante) exacte du gène de la nucléoprotéine $(\mathrm{N})$ appelé $\mathrm{N}-450$ pour l'épidémiologie moléculaire et la séquence entière du gène (1854 nt) de l'hémagglutinine $(H)$ pour obtenir des renseignements supplémentaires $(3,4)$. Puisque le génome de la rougeole mute très lentement, les cas relevant d'une même éclosion ou chaîne de transmission portent, habituellement, des séquences N-450 identiques. Les différences, même concernant un seul nucléotide, suffisent généralement pour exclure une transmission directe entre deux cas.

L'OMS tient à jour une base de données sur les séquences de la rougeole appelée MeaNS (5) (http://www.whomeasles.org/Public/Web_Front/sequence.php), dans laquelle les membres du Réseau mondial de laboratoires pour la rougeole (LabNet) de l'OMS soumettent des séquences (gènes $\mathrm{N}-450$ et $\mathrm{H}$ ) tirées de cas de rougeole. Les membres de LabNet sont également en mesure de comparer les séquences N-450 de leurs cas avec des séquences déposées par d'autres membres afin de trouver d'autres zones géographiques où la séquence de la rougeole a été détectée. Cette information peut fournir des preuves de laboratoire en vue d'appuyer ou d'exclure d'éventuels liens entre les cas importés et leur pays d'origine.

Pour les séquences N-450 couramment détectées, la base de données MeaNS désigne une "variante de la séquence » (3). Parmi les exemples de variantes de la séquence, nous pouvons citer la variante MVs/Manchester.GBR/10.09 (génotype D4) et la variante MVs/Taunton.GBR/27.12 (génotype D8). Les membres de LabNet consultent la base de données MeaNS pour déterminer à quelle variante de la séquence correspond la séquence de leur cas de rougeole. La variante de la séquence devient la caractéristique qui permet de définir des cas et des éclosions de rougeole. Elle est utilisée pour trouver facilement les séquences de la rougeole correspondantes issues d'autres cas éventuellement liés (p. ex. dans le cadre d'une même éclosion) ou de pays cibles.

Au Canada, le génotypage de la rougeole est effectué au Laboratoire national de microbiologie, un laboratoire régional de référence de l'OMS concernant la rougeole et la rubéole. Les laboratoires provinciaux sont invités à soumettre tous leurs échantillons de rougeole positifs et suspects (p. ex. des écouvillons de sécrétions nasopharyngées ou des échantillons d'urine) au Laboratoire national de microbiologie aux fins de génotypage. Bien que tous les cas de rougeole doivent être génotypés, seulement ceux avec des échantillons adéquats peuvent l'être en effet. Le Laboratoire national de microbiologie renvoie aux provinces les renseignements sur le génotypage de la rougeole (c.-à-d. le génotype, la désignation de la séquence et, le cas échéant, la désignation de la variante identique de la séquence). Les désignations des séquences sont les noms normalisés de l'OMS pour les séquences de la rougeole tirées des différents cas de la maladie. Ils servent à indiquer le lieu (la ville, la province, l'état et le pays) et la période (par semaine et année épidémiologique à partir de l'apparition de l'éruption cutanée ou de la collecte de l'échantillon) du cas de rougeole $(3,4)$. Par exemple, la MVs/Ontario.CAN/22.13 est une séquence de la rougeole tirée d'un cas en Ontario (Canada) au cours de la 
$22^{e}$ semaine épidémiologique de 2013. Le Laboratoire national de microbiologie signale également les cas à l'OMS en saisissant les séquences dans la base de données MeaNS.

\section{Utilité et nécessité de l'épidémiologie moléculaire de la rougeole}

Le Canada a subi un nombre important de cas et d'éclosions de rougeole en 2013, dont une partie a eu lieu simultanément dans différentes provinces (6). Dans certains cas, l'identification du génotype de la rougeole suffisait à elle seule pour vérifier que les éclosions simultanées étaient bien distinctes (p. ex. au début de 2013, des cas de rougeole de génotype B3 sont survenus en Ontario peu après l'apparition distincte de cas de génotype D8 dans la même province [6]). En revanche, la rougeole de génotype D8 a été détectée tout au long de l'année et dans presque toutes les provinces ayant signalé des cas de rougeole. De prime abord, cela pourrait être inquiétant pour un pays qui a éliminé la rougeole. L'analyse des données de la séquence a permis de démontrer que les cas de génotype D8 peuvent être davantage caractérisés en tant que quatre variantes de séquence différentes (6) et n'étaient donc pas dus à la propagation d'une seule variante.

En plus des analyses épidémiologiques globales, le génotypage de la rougeole peut fournir des renseignements utiles pendant les enquêtes en temps réel sur des cas de rougeole. En 2013, la rougeole a été importée par deux touristes canadiens qui s'étaient rendus indépendamment dans le même centre de villégiature au Mexique(6), un autre pays ayant éliminé la rougeole endémique (2). Grâce au génotypage et à l'utilisation de la base de données MeaNS, il a été possible d'indiquer le Royaume-Uni comme source possible du virus (Kevin Brown, Santé publique, Angleterre : communication personnelle du 7 mars 2013), ce qui a été par la suite confirmé par l'enquête épidémiologique (6).

Il convient de noter que l'épidémiologie moléculaire de la rougeole repose sur la collecte et l'envoi d'échantillons cliniques appropriés de tous les cas suspects de rougeole (écouvillons de sécrétions nasopharyngées ou échantillons d'urine; pour plus de détails, consulter la source [7] ainsi que le Guide des services du Laboratoire national de microbiologie disponible à l'adresse https://www.nml-Inm.gc.ca/guide2/index-fra.htm). Afin de faire le suivi de la réussite des programmes d'élimination de la rougeole, l'OMS recommande que LabNet procède au génotypage d'au moins $80 \%$ des cas et des éclosions sporadiques (c.-à-d. le génotypage d'au moins un cas de $\geq 80 \%$ des éclosions).

\section{Orientations futures}

L'OMS a ciblé la rougeole pour une éradication mondiale (8) et, à mesure que des progrès sont réalisés, la diversité génétique des virus de la rougeole diminue. Souvent, une seule variante de la séquence de la rougeole est responsable de grandes éclosions persistantes, en particulier dans les régions qui n'ont pas éliminé la maladie. Ces éclosions peuvent constituer des réservoirs pour l'importation de la rougeole au Canada. S'il y a plusieurs importations de la même variante, nous sommes incapables de les distinguer à l'aide des actuelles méthodes de génotypage de la rougeole. En 2011, la variante de séquence MVs/Manchester.GBR/10.09 de génotype D4 a été importée à plusieurs reprises au Québec, en Colombie-Britannique et en Ontario et était liée à la grande éclosion au Québec (voir la source [9] et des données non publiées). La capacité de l'épidémiologie moléculaire de démontrer l'absence de transmission endémique a été contestée. Il est, par conséquent, nécessaire d'étendre les méthodes de génotypage au-delà du gène $\mathrm{N}-450$ pour recueillir des données supplémentaires sur les séquences en vue de trouver des différences entre les cas de rougeole. En 2013, le Laboratoire national de microbiologie a mis en place un séquençage systématique du gène $\mathrm{H}$ pour tous les cas de rougeole. On pourrait distinguer à l'aide des séquences du gène $\mathrm{H}$ les différentes importations de la souche D8 à partir des Pays-Bas (identique à la variante de séquence MVs/Taunton.GBR/27.12), ainsi que les éclosions et les cas de rougeole non liés comprenant une seule souche de rougeole de génotype B3 (identique à la variante de séquence $\mathrm{MVi} / \mathrm{Harare} . \mathrm{ZWE} / 38.09)$, toutes deux différenciables au gène $\mathrm{N}-450$ (6). Cependant, comme le séquençage du gène $\mathrm{H}$ ne suffit pas toujours, le séquençage du génome en entier sera probablement nécessaire à mesure que l'on progresse dans l'élimination mondiale de la maladie. 


\section{Conclusion}

L'épidémiologie moléculaire de la rougeole est un outil précieux pour faire le suivi des importations, relier les cas et démontrer l'absence de transmission soutenue de la rougeole. À mesure que la diversité génétique des virus de la rougeole diminue, il deviendra nécessaire d'étendre le génotypage et, au bout du compte, d'effectuer le séquençage du génome en entier pour une surveillance moléculaire efficace. Le maintien d'une surveillance de haute qualité pour les cas de rougeole, qui comprend le génotypage (nécessitant la collecte d'échantillons appropriés), est essentiel au suivi de l'élimination durable de la rougeole au Canada.

\section{Références}

(1) Agence de la santé publique du Canada. Élimination de la rougeole, de la rubéole et du syndrome de rubéole congénitale au Canada. Documentation et rapport de vérification. Décembre 2011. Accès : http://www.phac-aspc.gc.ca/im/vpd-mev/measles-rougeole-mrer-fra.php

(2) Castillo-Solorzano CC, Matus CR, Flannery B, Marsigli C, Tambini G, Andrus JK. The Americas: paving the road toward global measles eradication. J Infect Dis. 2011; 204(Suppl 1):S270-8.

(3) Organisation mondiale de la Santé. Nomenclature des virus rougeoleux: mise à jour 2012. Relevé épidémiologique hebdomadaire. 2012; 9(87):73-80.

(4) Organisation mondiale de la Santé. Nomenclature relative à la description des caractéristiques génétiques des virus rougeoleux sauvages: standardisation. Relevé épidémiologique hebdomadaire. 1998; 73:265-272.

(5) Rota PA, Brown K, Mankertz A, Santibanez S, Shulga S, Muller CP, Hübschen JM, Siqueira M, Beirnes J, Ahmed H, Triki H, Al-Busaidy S, Dosseh A, Byabamazima C, Smit S, Akoua-Koffi C, Bwogi J, Bukenya H, Wairagkar N, Ramamurty N, Incomserb P, Pattamadilok S, Jee Y, Lim W, Xu W, Komase K, Takeda M, Tran T, Castillo-Solorzano C, Chenoweth P, Brown D, Mulders MN, Bellini WJ, Featherstone D. Global distribution of measles genotypes and measles molecular epidemiology. J Infect Dis. 2011; 204(Suppl 1), S514.

(6) Agence de la santé publique du Canada. La surveillance de la rougeole au Canada : Tendances 2013; CCDR Vol 40-12;244.

(7) Groupe de travail pour l'élimination de la rougeole et de la rubéole, Santé Canada, Agence de la santé publique du Canada. Lignes directrices pour la prévention et le contrôle des éclosions de rougeole au Canada. Relevé des maladies transmissibles au Canada. 2013; 39(DCC-3www.phac-aspc.gc.ca/publicat/ccdrrmtc/13vol39/acs-dcc-3/index-fra.php

(8) World Health Organization. Global eradication of measles: report by the Secretariat. Geneva, Switzerland: World Health Organization; 2010. http://apps.who.int/gb/ebwha/pdf_files/wha63/a63_18-en.pdf

(9) De Serres G, Markowski F, Toth E, Landry M, Auger D, Mercier M, Bélanger P, Turmel B, Arruda H, Boulianne N, Ward BJ, Skowronski DM. J Infect Dis. 2013; 207(6):990-8.

\section{Remerciements}

Les auteurs remercient Elizabeth McLachlan et Lillian Mendoza pour leur examen critique du manuscrit.

\section{Conflit d'intérêts}

II n'y a aucun conflit d'intérêts à déclarer. 